\section{gp130's sensitive side}

By Lauren Martz, Staff Writer

Medical University Innsbruck researchers suggest that inhibiting the gp130 pathway in peripheral sensory nerve cells could reduce certain types of pain. ${ }^{1}$ Coupled with previous findings that activation of the gp130 pathway on immune cells can lead to cancer and inflammation, the new research suggests that hitting the target on both types of cells could treat those diseases and reduce the pain associated with them.

Many cytokines activate immune cells by activating the IL- 6 signal transducer (IL-6ST; gp130; CD130) on the cell surface. ${ }^{2}$ When the pathway works properly, the activated immune cells are able to ward off infections. But when it goes awry, the result can be inflammatory conditions or cancer.

Hints that gp130 may have additional roles emerged in 2005, when Michaela Kress and colleagues showed that the target is also found on sensory neurons. ${ }^{3}$

Kress, a professor of physiology at Innsbruck who made the sensory neuron finding while at the University of Erlangen-Nurnberg, has now uncovered the therapeutic implications of that research.

First, her team developed conditional knockout mice with gp130 absent specifically in the nociceptors. In models of inflammatory and cancer pain, the conditional knockout mice had less hyperalgesia than wild-type mice.

Next, the team treated wild-type and knockout mice with a fusion protein of IL- 6 and its receptor. It was a logical choice, as the complex of IL-6 and its receptor is known to work through gp130 to cause inflammation.

gp130 knockout animals receiving the fusion protein had only slight increases in their pain response to heat, whereas wild-type mice receiving the fusion protein had a substantial rise in sensitivity. Treated wild-type mice also had increased levels of transient receptor potential vanilloid 1 (VR1; TRPV1), which is an ion channel that helps transduce nociceptor pain signals.

Collectively, the findings suggest that IL-6 signaling through gp130 is important for pain sensitivity and that VR1 is required for pain induced by IL-6. Results were published in The Journal of Neuroscience.

\section{Dual-ing with gp130}

Kress told SciBX that the next steps are to study the analgesic effects of gp130 neutralization through antibodies or soluble decoy receptors. The first indications will be ones in which her team thinks a gp130 inhibitor can have a dual effect by acting on both immune cells and peripheral neurons.

"gp130 has proproliferative effects in a number of cancer types, such as breast cancer and cancer of the pancreas," she said. "We expect that neu- tralization of gp130 would provide a dual benefit for certain cancer pain patients by a potential antiproliferation effect plus an analgesic effect."

Beth Habecker, associate professor of physiology and pharmacology at the Oregon Health \& Science University School of Medicine, agreed that cancer pain would be a good starting point.

She said a condition such as arthritis would require chronic use of a gp130 inhibitor, which could lead to resistance over time. Resistance in cancer should be less of an issue, she said.

Hwan Tae Park of Dong-A University College of Medicine thinks blocking gp130 could be well suited to inhibit pain in the periphery, such as at the sites of inflammation. Park, an associate professor in the Department of Physiology, also said blocking gp130 could produce better results than compounds that target IL-6.

"IL-6 may not be the sole hyperalgesic ligand for gp130, and because the definitive role of endogenous IL- 6 has not been revealed, it might be better to develop a gp130-binding antagonist rather than an IL-6 blocker," he said.

Jalal Jazayeri, lecturer in the Department of Pharmaceutical Biology at Monash University, also noted that "targeting gp130 could block the effects of more than one cytokine. It is like killing a few birds with one stone."

The downside of gp130's interaction with many upstream cytokines is that it could prove challenging to disrupt the target in pain, cancer or inflammation without affecting its role in the body's natural immune response. Indeed, because of its broad effects, there are no disclosed gp130 inhibitors in development.

"Blocking gp130 signaling may affect several physiological events in the body," noted Park.

"Total disruption of gp130 signaling is likely to cause as many problems as it resolves," said Habecker. "These could be addressed with a strategy that targets treatment to sensory nerves."

Park agreed that localizing a gp130 inhibitor to the inflammatory sites or tumor regions could circumvent systemic side effects.

Kress also agreed. He said his team is testing some strategies to target therapy to neurons, but they don't yet have any promising strategies for targeting cancer cells.

Kress told SciBX his team has not yet filed a patent application on the findings.

Martz, L. SciBX 2(45); doi:10.1038/scibx.2009.1651

Published online Nov. 19, 2009

\section{REFERENCES}

1. Andratsch, M. et al. J. Neurosci.; published online Oct. 28, 2009; doi:10.1523/JNEUROSCI.1822-09.2009

Contact: Michaela Kress, Medical University Innsbruck, Innsbruck, Austria

e-mail: michaela.kress@i-med.ac.at

2. Yoshida, K. et al. Proc. Natl. Acad. Sci. USA 93 407-411 (1996)

3. Obreja, O. et al. Brain 128 1634-1641 (2005)

\section{COMPANIES AND INSTITUTIONS MENTIONED}

Dong-A University College of Medicine, Busan, South Korea

Medical University Innsbruck, Innsbruck, Australia

Monash University, Melbourne, Victoria, Australia

Oregon Health \& Science University School of Medicine, Portland, Ore.

University of Erlangen-Nurnberg, Erlangen, Germany 